\title{
Integrative Review on Cognitive Behavioral Therapy in Chronic Diseases: The Responses Predictors
}

\author{
Nada Lukkahatai ${ }^{1 *}$, Jillian Inouye ${ }^{2}$, Diane Thomason $^{3}$, Jennifer Kawi ${ }^{4}$, Bruce Leonard ${ }^{5}$ and Kirsten \\ Connelly ${ }^{4}$
}

${ }^{1}$ Department of Nursing, Johns Hopkins University, Baltimore, USA

${ }^{2}$ Department of Medicine, University of Hawaii at Manoa, USA

${ }^{3}$ Department of Nursing, Arizona College, USA

${ }^{4}$ Department of Nursing, University of Nevada, USA

${ }^{5}$ Department of Nursing, University of Texas Medical Branch, USA

*Corresponding author: Nada Lukkahatai, Department of Nursing, Johns Hopkins University, USA.

Received Date: May 22, 2019

Published Date: June 11, 2019

\begin{abstract}
Background: Cognitive behavioral therapy (CBT) is a self-management strategy used by patients with chronic diseases. Studies consistently report the effectiveness of this therapy in managing symptoms and improving patients' quality of life. However, evidence also shows that not all patients benefit from the therapy.
\end{abstract}

Methods: This article presents findings from an integrative review of studies published between 2010 and 2014 that investigated outcome predictors of CBT in chronic illness. The use of CBT in psychological disorders was excluded from the review.

Result: Eleven studies were included into this review. Every study supported the effectiveness of CBT for both immediate and long-term outcomes. The intervention components of CBT used in these studies were varied in the number and duration of sessions and the methods of identifying the effectiveness of the CBT. Most studies investigated the significant predictability of the psychological variables. Only one study investigated physiological predictors, and none investigated biological predictors.

Conclusion: This result highlighted the importance of consistency in the CBT components and methods used to identify the effectiveness of therapy. Furthermore, including physical and biological predictors of CBT outcomes is warranted, specifically in patients with a chronic illness.

Keywords: Cognitive behavioral therapy; Outcome predictors; Chronic diseases

\section{Introduction}

Chronic illnesses are rapidly becoming a major health concern in the United States. Over half of the adult population is reported to have at least one major chronic condition. Chronic illnesses often cause permanent and irreversible physiological changes that impact the individual's physical, psychological, social, and economic status. Chronic conditions are associated with substantial disability and considerable health care cost [1]. Despite differences in disease etiology, people living with chronic illnesses encounter similar diseases management challenges. These challenges include adjusting their lifestyle, dealing with emotion and psychological responses to chronic illnesses, identifying associated symptoms, and adhering to a medication regimen [2]. While there are many self-management strategies or ways to improve self-care activities and optimize health while living with a chronic illness, cognitive behavioral therapy (CBT) is one that shows evidence of good outcomes.

Cognitive-behavioral therapy is a biopsychosocial intervention that combines techniques such as cognitive restructuring, relaxation, problem-solving, and stress management [3]. The underlying concept of CBT is an appraisal of individual behavioral responses to ways of thinking, mood expression, physical symptoms, and behavioral responses to an event or events [4]. Therefore, the goals 
of CBT focus on challenging cognitive distortions and dysfunctional underlying beliefs and teaching coping and problem-solving skills [5]. To achieve cognitive and behavioral changes, the individual must actively participate in a collaborative problem-solving process and modify maladaptive behavioral patterns. The overall outcomes of CBT include symptom reduction, improvement of function, disease control, and an improved quality of life [6-8].

Since CBT was developed in 1995, it has been extensively used for the treatment of psychological conditions. It has also been found to have potential benefits to persons with chronic physical illnesses who cannot adjust to the disease, or beliefs and behaviors related to it. Cognitive behavioral therapy has been used in studies of people with cancer Thomas \& Weiss [9], Parkinson's diseases Dobkin et al. [10], diabetes Welschen et al. [11], human immunodeficiency Inouye, Flannelly, Flannelly, Wagner et al. [12], fibromyalgia and arthritis V. G. Sinclair \& Wallston [13], and diabetes K. A. Sinclair et al. $[14,15]$.

Several studies report that CBT:

a) Improved mood problems such as anxiety and depression.

b) Changed disease-specific beliefs and attitudes.

c) Improved psychological and physiological outcomes and.

d) Changed health behaviors such as medication adherence and improved quality of life [16-18].

Outcome measurements for these studies included symptom reduction [19-21], enhanced physical function [22], and improved psychological conditions, including depression, anxiety, and fear [23]. The similarity in implementing CBT for a variety of chronic diseases is that it is delivered by clinicians or healthcare professionals with a masters-level education or higher, including nurses and psychologists.

Notall studies report that patients who receive CBT demonstrate improved outcomes. Systematic reviews have reported inconsistent findings on the effectiveness of CBT on physical outcomes, such as pain, fatigue, and sleep [24,25]. A review of randomized control trails on the self- management of chronic illness found that CBT was an effective strategy and increased self- efficacy, improved moods and coping ability, and improved the quality of life in Asians and Pacific Islanders living with chronic illnesses [26]. Based on variable outcomes in studies of $\mathrm{CBT}$, investigators have begun to examine predictors of treatment success.

Systematic reviews and meta-analyses have reported the effectiveness of CBT and predictors of treatment outcomes in different psychological disorders, including schizophrenia, bipolar disorder, major depression, anxiety disorder, eating disorders, and obesity $[27,28]$. Fewer studies have investigated the effectiveness of CBT in physiological illnesses such as cancer, fibromyalgia, arthritis, chronic pain, diabetes, and HIV [29]. One review article included the outcome predictors as part of the review of behavioral and cognitive-behavioral treatment in persons with chronic pain McCracken \& Turk [30]. These authors reviewed studies published between 1989 and 1999 using both behavioral treatment and CBT but limited their search to a population with chronic pain. A more recent systematic review published in 2013 reported the predictors of treatment outcomes for patients with fibromyalgia de Rooij et al. [31]. Although they found that the level of depression, belief, disability, and pain were predictors of treatment outcomes, the treatment used in this review was not specific for CBT. The purpose of this paper is to review the predictors of outcomes of CBT intervention among the people with chronic diseases.

\section{Methods}

\section{Study selection}

We searched PubMed, PsycINFO, SCOPUS, and EMBASE for articles published between 2010 and 2014 that included clinical trials of adults aged 18 years and older, published in English, and with the following keywords as all fields: "Cognitive Behavioral Therapy" OR "Cognitive Behavioral Intervention" AND "Predictor." The search yielded 3,701 articles, but the removal of duplicates left 2,999 . To investigate the use of CBT in chronic physical illnesses, these studies were then screened by title to remove those that focused on psychological disorders and weight control. The refined search yielded 607 articles. Abstracts from these articles were reviewed to determine if they met the final inclusion criterion of including the predictors of the cognitive behavioral intervention. Ninety-eight articles remained after the abstract review. Finally, the full text of the 98 articles was reviewed for inclusion of the predictors of CBT effectiveness. Eleven articles met the criteria and were included in this review (Figure 1).

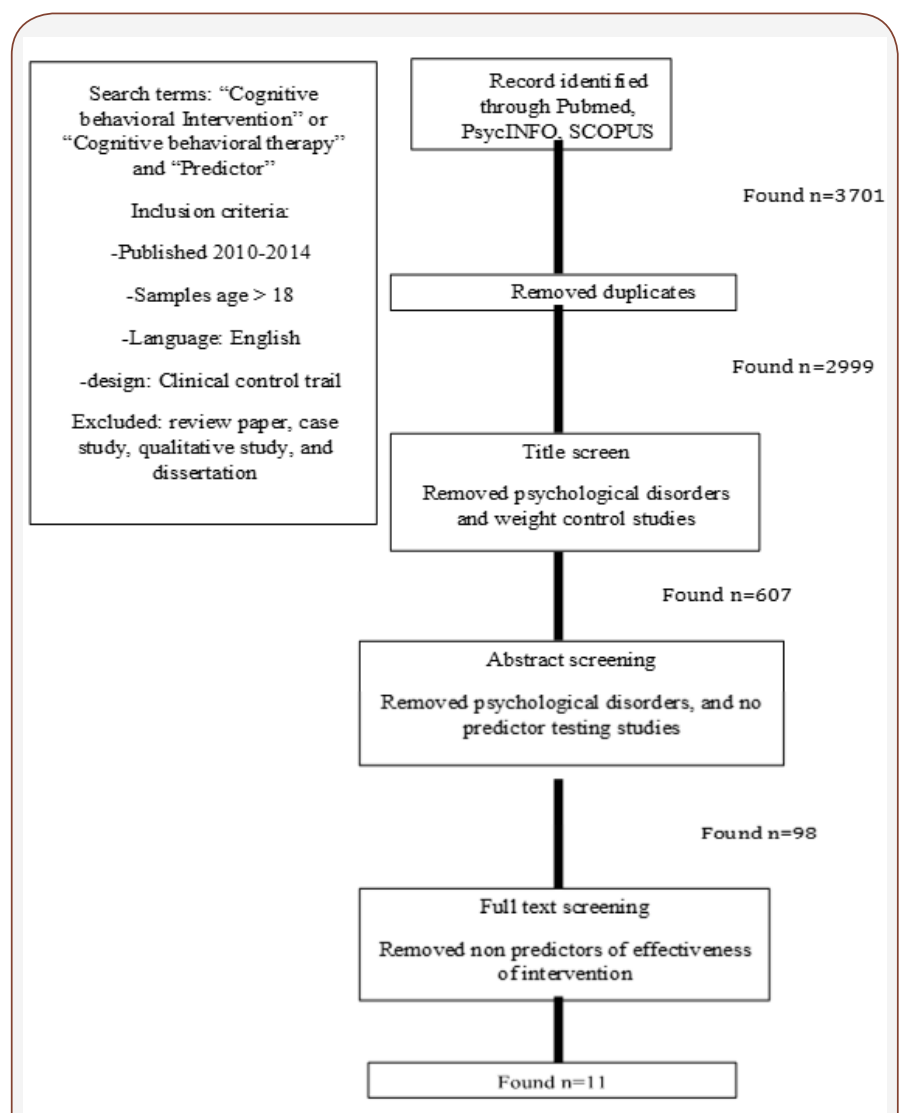

Figure 1: Flow chart of literature search process. 


\section{Quality assessment}

Four reviewers independently evaluated the quality of 11 studies using the Jadad Scoring of Quality of Reports of Randomized Clinical Trials instrument Jadad, Carroll, Moore, \& McQuay [32]. This is a validated instrument used to evaluate the quality of randomized clinical trials. It emphasizes specific parts of a study, including randomization, blinding, withdrawal, and dropouts. It is an 11-item assessment the reviewer uses to evaluate the quality of a study based on the description of the study and its methodology.
Each item is rated either $0=$ does not describe, or $1=$ describe. Two extra points can be added if the methods of randomization and a double-blind are described. Therefore, the total Jadad quality score ranges from 0 to 13 with the higher score indicating better quality. Of the 11 articles reviewed, 6 reported the details of their intervention and methodology in the original studies. Therefore, the reviewers evaluated the quality of these six articles based on the descriptions in the original studies [33,34]. The reviewers discussed the item scores among themselves until they came to a consensus (Table 1).

Table 1: Study characteristics.

\begin{tabular}{|c|c|c|c|c|c|c|}
\hline \multirow{2}{*}{ Authors (year) } & \multirow{2}{*}{ Study location } & \multirow{2}{*}{ Jadad score } & \multicolumn{3}{|c|}{ Sample Characteristics } & \multirow{2}{*}{ Chronic conditions } \\
\hline & & & Age $($ mean $\pm \mathrm{SD})$ & Gender, n (\%) & Race, n (\%) & \\
\hline $\begin{array}{l}\text { Chader, Godfrey, Ridsdale, } \\
\text { King and Wessely }\end{array}$ & $\begin{array}{c}\text { Primary care in } \\
\text { London and the South } \\
\text { East of England }\end{array}$ & 10 & $39.4 \pm 13.6$ & $\begin{array}{c}\text { Male: } 43(27 \%) \\
\text { Female: } 117(73 \%) \\
n=160\end{array}$ & NA & $\begin{array}{l}\text { Chronic fatigue } \\
\text { syndrome }\end{array}$ \\
\hline Currie, Wilson and Curran & $\begin{array}{l}\text { Outpatients from } \\
\text { chronic pain } \\
\text { treatment clinics }\end{array}$ & 8 & $45.0 \pm 8.0$ & $\begin{array}{l}\text { Male: } 27(45 \%) \\
\text { Female: } 33(55 \%) \\
\quad n=60\end{array}$ & NA & $\begin{array}{l}\text { Chronic nonmalignant } \\
\text { pain with insomnia }\end{array}$ \\
\hline Flor and Birbaumer & $\begin{array}{l}\text { Outpatients at the } \\
\text { psychophysiological } \\
\text { pain clinic }\end{array}$ & 7 & $42.4 \pm 9.7$ & $\begin{array}{c}\text { Male: } 31(40 \%) \\
\text { Female: } 47(60 \%) \\
\mathrm{n}=78\end{array}$ & White: $100 \%$ & $\begin{array}{c}\text { Chronic } \\
\text { musculoskeletal pain }\end{array}$ \\
\hline Gersh, et al. [38] & $\begin{array}{c}\text { Outpatients from pain } \\
\text { center at Victoria, } \\
\text { Australia }\end{array}$ & 5 & $49.5 \pm 14.1$ & $\begin{array}{c}\text { Male: } 97(37 \%) \\
\text { Female: } 164(63 \%) \\
n=261\end{array}$ & NA & $\begin{array}{l}\text { Chronic nonmalignant } \\
\text { pain }\end{array}$ \\
\hline Kempke, et al. [35] & $\begin{array}{c}\text { Patients from tertiary } \\
\text { care centre for CFS in } \\
\text { Belgium }\end{array}$ & 3 & $40.4 \pm 9.6$ & $\begin{array}{c}\text { Male: } 27(15 \%) \\
\text { Female: } 151(85 \%) \\
n=178\end{array}$ & NA & $\begin{array}{l}\text { Chronic fatigue } \\
\text { syndrome }\end{array}$ \\
\hline Litt \& Porto [37] & $\begin{array}{l}\text { Outpatients from } \\
\text { dental clinic in } \\
\text { Connecticut, USA }\end{array}$ & 7 & $39.4 \pm 12.1$ & $\begin{array}{c}\text { Male: } 16(16 \%) \\
\text { Female: } 85(84 \%) \\
\text { n=101 }\end{array}$ & $\begin{array}{l}\text { White: } 76 \% \\
\text { Black: } 9 \% \\
\text { Hispanic } \\
\text { origin: } 9 \% \\
\end{array}$ & $\begin{array}{c}\text { Temporomandibular } \\
\text { disorder (TMD) pain } \\
\text { patients }\end{array}$ \\
\hline Ljotsson, et al. [51] & $\begin{array}{c}\text { Outpatients } \\
\text { Stockholm, Sweden }\end{array}$ & 7 & $34.3 \pm 9.3$ & $\begin{array}{c}\text { Male: } 12(15 \%) \\
\text { Female: } 67(85 \%) \\
n=79\end{array}$ & & $\begin{array}{l}\text { Irritable bowel } \\
\text { syndrome (IBS) }\end{array}$ \\
\hline Prins, et al. & $\begin{array}{c}\text { Outpatients from } \\
\text { department of } \\
\text { internal medicine of } 2 \\
\text { universities }\end{array}$ & 9 & $36.7 \pm 10.1$ & $\begin{array}{c}\text { Male:58 }(21 \%) \\
\text { Female: } 212(79 \%) \\
n=270\end{array}$ & $\mathrm{~N} / \mathrm{A}$ & $\begin{array}{l}\text { Chronic fatigue } \\
\text { syndrome }\end{array}$ \\
\hline
\end{tabular}

a Information was published in Ridsdale, et al.

b Information was published in Currie, Wilson, Pontefract \& deLaplante 2000.

d Information was published in Zonneveld, van Rood, Timman, et al.

\section{Results}

Of the 11 articles evaluated, 9 (82\%) were in an outpatient setting. Only two studies (18\%) were done with inpatients receiving treatment at a tertiary rehabilitation center. The participants' ages ranged from 34 to 65 years. The number of participants in each study varied from 13 to 261 and in 9 studies, the majority was female, ranging from 62 to 88\%. Most of the studies in Europe and Australia did not report race or ethnicity, Studies conducted in the United States, however, reported a majority of white/Caucasians (76 to 93\%). Clinical populations investigated in the 11 articles had chronic nonmalignant pain, such as temporomandibular disorder, chronic low back pain ( $\mathrm{n}=4$ articles, 36\%), chronic fatigue syndrome ( $\mathrm{n}=2$ articles, $18 \%$ ), irritable bowel syndrome ( $\mathrm{n}=1$ article, 10\%), posttraumatic stress disorders in cancer survivor and civilian trauma ( $\mathrm{n}=2$ articles, 18\%), Parkinson's disease ( $\mathrm{n}=$
1 article, 10\%), and unexplained physical symptoms ( $\mathrm{n}=1$ article, $10 \%$ ). (Table 1) summarizes the characteristics of studies used in this review paper. The quality of the 11 articles based on the Jadad score ranged from 3 to 11.

\section{Intervention implementation}

Cognitive behavioral interventions used in the 11 articles (Table 2) varied in terms of the CBT features of treatment modality, delivery methods, and format. Several reviewed articles indicated that detailed information of their CBT intervention was published elsewhere. Therefore, the original articles were reviewed except for one study Kempke et al. [35], which was referenced in a nonpublished paper. The cognitive restructuring was the key CBT feature used in eight of the studies. Only two studies included relapse prevention (18\%) and three included the homework/ workbook requirement (27\%). Relative to treatment modality, 
four studies (36\%) evaluated the effectiveness of CBT as a single intervention, while the majority used CBT as an adjunct intervention ( $\mathrm{n}=7,64 \%$ ). CBT was primarily delivered in a face-to-face format ( $n=9,82 \%)$ with individual participants $(n=6,55 \%)$. Two studies used either the telephone or internet (Table 3). The length of an intervention varied from 1 to 5 hours per session and the number of sessions ranged from 6 to 75 . The most common length a session was 60 to 90 minutes $(n=4,36 \%)$ with 10 sessions $(n=5,45 \%)$ to complete the study (Table 3).

Table 2: Study characteristics.

\begin{tabular}{|c|c|c|c|c|c|c|c|}
\hline \multirow{2}{*}{ Authors } & \multirow{2}{*}{ Intervention (s) } & \multirow{2}{*}{$\begin{array}{c}\text { Theoretical } \\
\text { bases/focus of } \\
\text { intervention }\end{array}$} & \multirow{2}{*}{ CBT components } & \multicolumn{3}{|c|}{ CBT Delivering methods } & \multirow{2}{*}{$\begin{array}{l}\text { Treatment } \\
\text { fidelity }\end{array}$} \\
\hline & & & & Method & Type & Therapist & \\
\hline $\begin{array}{l}\text { Chalder, } \\
\text { Godfrey, } \\
\text { Ridsdale, } \\
\text { King and } \\
\text { Wessely }\end{array}$ & $\begin{array}{c}\text { Comparing } \\
\text { between } \\
\text { Counseling with } \\
\text { psychodynamic } \\
\text { approach } \\
\text { and Cognitive } \\
\text { behavioral therapy } \\
\text { (CBT) } \\
\end{array}$ & & $\begin{array}{l}\text { providing treatment } \\
\text { rationale activity } \\
\text { planning home work }\end{array}$ & $\begin{array}{l}\text { Face to face } \mathrm{Six} \\
\text { of } 1 \text {-h sessions }\end{array}$ & N/A & $\begin{array}{l}\text { Qualified CBT } \\
\text { therapists }\end{array}$ & $\begin{array}{l}\text { The session } \\
\text { were rated by } \\
4 \text { independent } \\
\text { CBT specialists }\end{array}$ \\
\hline $\begin{array}{l}\text { Currie, } \\
\text { Wilson and } \\
\text { Curran }\end{array}$ & $\begin{array}{l}\text { Cognitive } \\
\text { behavioral } \\
\text { Treatment of } \\
\text { insomnia }\end{array}$ & $\begin{array}{l}\text { The development of } \\
\text { insomnia secondary } \\
\text { to chronic pain }\end{array}$ & $\begin{array}{c}\text { Basic education } \\
\text { Behavioral therapy } \\
\text { targeted sleep } \\
\text { problem Relaxation } \\
\text { training Sleep hygiene } \\
\text { education }\end{array}$ & $\begin{array}{l}\text { Face to face } \\
\text { Seven 2-h } \\
\text { session }\end{array}$ & $\begin{array}{l}\text { Group (5-7 } \\
\text { participants) }\end{array}$ & $\begin{array}{l}\text { Doctoral } \\
\text { students } \\
\text { or interns } \\
\text { in clinical } \\
\text { psychology, } \\
\text { who had } \\
\text { previous } \\
\text { training of } \\
\text { CBT } \\
\end{array}$ & $\begin{array}{l}\text { Therapists } \\
\text { were required } \\
\text { to follow the } \\
\text { writing manual } \\
\text { and were } \\
\text { supervised } \\
\text { weekly }\end{array}$ \\
\hline $\begin{array}{l}\text { Flor and } \\
\text { Birbaumer }\end{array}$ & $\begin{array}{c}\text { Cognitive- } \\
\text { behavioral } \\
\text { therapy (CBT) vs. } \\
\text { electromyographic } \\
\text { biofeedback } \\
\text { (EMG-BFB) vs. } \\
\text { conservative } \\
\text { medical treatment } \\
\text { (MED) }\end{array}$ & $\begin{array}{l}\text { Patients with } \\
\text { psychophysiological } \\
\text { reactivity would } \\
\text { be benefit from } \\
\text { EMG- BFB. Patients } \\
\text { with negative self- } \\
\text { statement would be } \\
\text { benefit from the CBT }\end{array}$ & $\begin{array}{c}\text { Progressive } \\
\text { muscle relaxation } \\
\text { identification of pain } \\
\text { and tension-eliciting } \\
\text { events relaxation } \\
\text { exercise problem } \\
\text { solving practice } \\
\text { pain coping skill } \\
\text { (e.g., positive self } \\
\text { statement,distraction) }\end{array}$ & $\begin{array}{l}\text { Face to face } \\
\text { Eight 1-h } \\
\text { sessions }\end{array}$ & N/A & $\begin{array}{l}\text { Clinical } \\
\text { psychologists } \\
\text { trained in } \\
\text { psychological } \\
\text { pain } \\
\text { treatment }\end{array}$ & N/A \\
\hline $\begin{array}{l}\text { Gersh et al. } \\
\quad[38]\end{array}$ & $\begin{array}{c}\text { Multidisciplinary } \\
\text { pain management } \\
\text { program included } \\
\text { Cognitive } \\
\text { behavioral } \\
\text { therapy (CBT) } \\
\text { and functional } \\
\text { restoration group } \\
\text { (FRG) }\end{array}$ & & $\begin{array}{c}\text { Modify the } \\
\text { maladaptive beliefs } \\
\text { and attitudes } \\
\text { about pain New } \\
\text { coping strategies } \\
\text { Relaxation Stress } \\
\text { reductiontechniques } \\
\text { Goal setting } \\
\text { Planned behavioral } \\
\text { reactivation Pacing } \\
\text { of physical activity } \\
\text { Guided gentle exercise } \\
\text { with Feldenkrias, tai } \\
\text { chi or individualized } \\
\text { gym program }\end{array}$ & $\begin{array}{l}\text { Face-to-face } \\
8 \text { full day } \\
\text { sessions } \\
\text { twice a week } \\
\text { (5 hours of } \\
\text { interactive } \\
\text { participation) }\end{array}$ & Group & $\begin{array}{l}\text { Trained } \\
\text { psychologist } \\
\text { in pain } \\
\text { management }\end{array}$ & N/A \\
\hline $\begin{array}{l}\text { Kempke et } \\
\text { al. [35] }\end{array}$ & $\begin{array}{c}\text { Cognitive- } \\
\text { behavioral based } \\
\text { Multi-component } \\
\text { treatment } \\
\text { program } \\
\text { Combination } \\
\text { of the group } \\
\text { CBT, flexible } \\
\text { graded exercise } \\
\text { therapy (GET) } \\
\text { and progressive } \\
\text { relaxation therapy } \\
\text { (PRT) }\end{array}$ & $\begin{array}{l}\text { Cognitive- behavioral } \\
\text { model (Vercoulen et } \\
\text { al., 1998) }\end{array}$ & N/A & Face-to-face & Group & N/A & N/A \\
\hline
\end{tabular}




\begin{tabular}{|c|c|c|c|c|c|c|c|}
\hline $\begin{array}{l}\text { Litt \& Porto } \\
\text { [37] }\end{array}$ & $\begin{array}{c}\text { Combination } \\
\text { of Standard } \\
\text { conservative } \\
\text { care (STD) } \\
\text { and Cognitive } \\
\text { behavioral therapy } \\
\text { (CBT) }\end{array}$ & & $\begin{array}{l}\text { Briefs cognitive- } \\
\text { behavioral program } \\
\text { include Relaxation } \\
\text { training } \\
\text { Stress management } \\
\text { Cognitive } \\
\text { restructuring }\end{array}$ & $\begin{array}{l}\text { Face to face } \\
6 \text { sessions } \\
\text { over 6-9 week } \\
\text { period }\end{array}$ & $\mathrm{N} / \mathrm{A}$ & $\begin{array}{l}\text { Four master } \\
\text { - level } \\
\text { therapists } \\
\text { with at } \\
\text { least } 2 \text { year } \\
\text { experience } \\
\text { in CBT with } \\
\text { medical } \\
\text { patients. }\end{array}$ & $\begin{array}{l}\text { Treatment } \\
\text { sessions were } \\
\text { videotaped and } \\
\text { reviewed by } \\
\text { first author }\end{array}$ \\
\hline $\begin{array}{l}\text { Ljotsson et } \\
\text { al., [53] }\end{array}$ & $\begin{array}{l}\text { Internet-delivered } \\
\text { cognitive } \\
\text { behavioral } \\
\text { treatment based } \\
\text { on exposure } \\
\text { exercises (ICBT) }\end{array}$ & $\begin{array}{l}\text { The principle of } \\
\text { behavioral exposure } \\
\text { and mindfulness } \\
\text { exercise }\end{array}$ & $\begin{array}{l}\text { Rational and } \\
\text { instructions on } \\
\text { mindfulness Identify } \\
\text { the thoughts and } \\
\text { symptoms using } \\
\text { mindfulness practice } \\
\text { Exposure exercises } \\
\text { include 1) Engaging in } \\
\text { activities that provoke } \\
\text { symptoms } \\
\text { 2) Establish behaviors } \\
\text { controlling symptoms } \\
\text { 3) Exposure to } \\
\text { situations where } \\
\text { symptoms are } \\
\text { unwanted. }\end{array}$ & $\begin{array}{c}\text { Internet } \\
\text { Administering } \\
\text { online (10 } \\
\text { weeks) }\end{array}$ & Individual & $\begin{array}{l}\text { Graduate } \\
\text { psychology } \\
\text { student } \\
\text { trained in } \\
\text { CBT respond } \\
\text { to online } \\
\text { synchronous } \\
\text { message } \\
\text { system from } \\
\text { participants }\end{array}$ & $\begin{array}{c}\text { No therapist } \\
\text { adherence to } \\
\text { the manual } \\
\text { was tested. All } \\
\text { participant } \\
\text { contact } \\
\text { monitored and } \\
\text { supervised. }\end{array}$ \\
\hline Prins, et al. & $\begin{array}{l}\text { Cognitive } \\
\text { behavioral therapy } \\
\text { and support group }\end{array}$ & $\begin{array}{l}\text { The model of CFS } \\
\text { focus on bodily } \\
\text { symptoms. The CBT } \\
\text { is directed at the } \\
\text { low sense of control, } \\
\text { low physical activity } \\
\text { and functional } \\
\text { impairment }\end{array}$ & $\begin{array}{l}\text { Explain the model of } \\
\text { CFS and perpetuate } \\
\text { factors Cognitive } \\
\text { restructuring to } \\
\text { improve sense } \\
\text { of control over } \\
\text { symptoms and } \\
\text { facilitate the behavior } \\
\text { change Maintain } \\
\text { physical activity, } \\
\text { gradually increase } \\
\text { physical activity } \\
\text { relapse prevention }\end{array}$ & $\begin{array}{l}\text { Face to face } \\
16 \text { sessions of } \\
\text { 1-hour CBT } \\
\text { over } 8 \text { months }\end{array}$ & Individual & $\begin{array}{l}\text { CBT trained } \\
\text { behavior } \\
\text { therapists } \\
\text { (include } \\
\text { psychologist, } \\
\text { psychiatrists } \\
\text { and health } \\
\text { scientists) }\end{array}$ & $\begin{array}{l}\text { Therapists } \\
\text { were } \\
\text { supervised } \\
\text { once every } \\
2 \text { weeks. } \\
\text { Audiotaped } \\
\text { CBT sessions } \\
\text { were randomly } \\
\text { checked to } \\
\text { ensure the } \\
\text { integrity of the } \\
\text { intervention. }\end{array}$ \\
\hline $\begin{array}{l}\text { Samwel et } \\
\text { al., [39] }\end{array}$ & $\begin{array}{l}\text { Multidisciplinary } \\
\text { allocation of } \\
\text { chronic pain } \\
\text { treatment. The } \\
\text { modalities include } \\
\text { 1) Medical } \\
\text { treatment } \\
\text { 2) Self adjusted } \\
\text { Transcultaneous } \\
\text { electrical nerve } \\
\text { stimulation } \\
\text { (TENS) } \\
\text { 3) Cognitive- } \\
\text { behavioral group } \\
\text { therapy }\end{array}$ & $\begin{array}{l}\text { Multidisciplinary } \\
\text { approaches }\end{array}$ & $\begin{array}{l}\text { Cognitive behavioral } \\
\text { group therapy was } \\
\text { selected if pain was } \\
\text { not reduced by both } \\
\text { medication and } \\
\text { TENS. Treatment } \\
\text { components included } \\
\text { Goal setting Stress } \\
\text { management } \\
\text { Problem-solving } \\
\text { techniques Cognitive } \\
\text { therapy Relaxation } \\
\text { exercise }\end{array}$ & $\begin{array}{c}\text { Face-to-face } \\
\text { Ten } 90 \text {-minute } \\
\text { sessions Group } \\
\text { of } 5 \text { to } 12 \\
\text { patients CBT }\end{array}$ & Group & Psychologist & \\
\hline $\begin{array}{l}\text { Schreurs, et } \\
\text { al. [41] }\end{array}$ & $\begin{array}{l}\text { Multidisciplinary } \\
\text { rehabilitation } \\
\text { program: } \\
\text { Combination of the } \\
\text { Graded exercise } \\
\text { therapy (GET) } \\
\text { and Cognitive } \\
\text { behavioral therapy } \\
\text { (CBT) }\end{array}$ & $\begin{array}{l}\text { Evidence based } \\
\text { treatments }\end{array}$ & $\begin{array}{l}\text { CBT components } \\
\text { include } \\
\text { Cognitive } \\
\text { restructuring Problem } \\
\text { solving Individual goal } \\
\text { setting }\end{array}$ & $\begin{array}{l}\text { Face to face } \\
75 \text { therapy } \\
\text { sessions } \\
\text { within } 25 \\
\text { weeks }\end{array}$ & Group & $\begin{array}{l}\text { Psychologists } \\
\text { and social } \\
\text { workers }\end{array}$ & \\
\hline
\end{tabular}




\begin{tabular}{|c|c|c|c|c|c|c|c|}
\hline $\begin{array}{l}\text { Sharpe, } \\
\text { et al. }\end{array}$ & $\begin{array}{l}\text { Combination } \\
\text { of medical care } \\
\text { and cognitive } \\
\text { behavioral therapy }\end{array}$ & $\begin{array}{l}\text { The intervention } \\
\text { is based on the } \\
\text { hypothesis that } \\
\text { factors such as } \\
\text { beliefs, coping } \\
\text { behavior, mood, } \\
\text { social and } \\
\text { pathophysiological } \\
\text { process are } \\
\text { interacted to impact } \\
\text { the illness }\end{array}$ & $\begin{array}{l}\text { Identify the illness } \\
\text { perception, and } \\
\text { consider the } \\
\text { psychological and } \\
\text { social factors that } \\
\text { influences the } \\
\text { illness Gradual and } \\
\text { consistent increase } \\
\text { in activity and } \\
\text { evaluation of the } \\
\text { strategy excessive } \\
\text { perfectionism } \\
\text { reduction active } \\
\text { problem solving } \\
\text { to interpersonal } \\
\text { and occupational } \\
\text { difficulties }\end{array}$ & $\begin{array}{c}\text { Face to face } \\
16 \text { one-hour } \\
\text { sessions over } 4 \\
\text { months. }\end{array}$ & Individual & $\begin{array}{c}\text { Three expert } \\
\text { therapists }\end{array}$ & $\begin{array}{c}\text { Therapy was } \\
\text { codified in a } \\
\text { manual and } \\
\text { supervised } \\
\text { by an expert } \\
\text { experienced } \\
\text { cognitive } \\
\text { therapist. }\end{array}$ \\
\hline $\begin{array}{l}\text { Siemonsma, } \\
\text { et al. [42] }\end{array}$ & $\begin{array}{l}\text { Combination of } \\
\text { grade activity, } \\
\text { gradual exposure } \\
\text { in vivo, and } \\
\text { cognitive } \\
\text { treatment of } \\
\text { illness perceptions } \\
\text { (CTIP) }\end{array}$ & $\begin{array}{l}\text { Evidence-based } \\
\text { methods of } \\
\text { cognitive behavioral } \\
\text { treatment (CBT) in } \\
\text { rehabilitation }\end{array}$ & $\begin{array}{l}\text { Identify illness } \\
\text { perceptions and } \\
\text { maladaptive } \\
\text { illness perception } \\
\text { Create question } \\
\text { about maladaptive } \\
\text { illness perceptions } \\
\text { Formulate and test } \\
\text { alternative illness } \\
\text { perceptions Apply } \\
\text { and practice alterative } \\
\text { illness perceptions }\end{array}$ & $\begin{array}{c}\text { Face to } \\
\text { face 10-14 } \\
\text { of 1-hour } \\
\text { individual } \\
\text { treatment } \\
\text { sessions }\end{array}$ & Individual & $\begin{array}{l}\text { Physical } \\
\text { therapists, } \\
\text { occupational } \\
\text { therapists or } \\
\text { psychologists } \\
\text { received } 21 \\
\text { hours training } \\
\text { with } 20 \text { hours } \\
\text { refresher } \\
\text { course. }\end{array}$ & $\begin{array}{l}\text { Therapists } \\
\text { required } \\
\text { discussing } \\
\text { the progress } \\
\text { of each } \\
\text { patient with } \\
\text { psychologist } \\
\text { supervisor at } \\
\text { least twice. }\end{array}$ \\
\hline $\begin{array}{l}\text { Zonneveld, } \\
\text { et al., [52] }\end{array}$ & $\begin{array}{c}\text { Cognitive } \\
\text { behavioral group } \\
\text { training }\end{array}$ & $\begin{array}{l}\text { The consequence } \\
\text { model (Zonneveld, et } \\
\text { al., 2012b) }\end{array}$ & $\begin{array}{l}\text { Psychoeducation } \\
\text { Response prevention } \\
\text { Pacing activity Graded } \\
\text { activity and exercise } \\
\text { Problem solving } \\
\text { Breath and relaxation } \\
\text { exercise Cognitive } \\
\text { intervention using the } \\
\text { Ellis' ABC worksheet } \\
\text { Relapse prevention }\end{array}$ & $\begin{array}{c}\text { Face to face } \\
13 \text { weekly of } 2 \\
\text { hours session }\end{array}$ & Group & $\begin{array}{c}\text { Psychologists } \\
\text { with master's } \\
\text { degree and } \\
>3 \text { years } \\
\text { postmaster } \\
\text { experience } \\
\text { with group } \\
\text { therapy and/ } \\
\text { or group CBT } \\
\text { or trained } \\
\text { with first } \\
\text { author }\end{array}$ & \\
\hline
\end{tabular}

a intervention information was published in Ridsdale, et al. 2001.

e intervention information was published in Zonneveld, van Rood, Timman., et al. 2012.

Table 3: Intervention Features, Treatment Modality and Delivery Methods, and Format.

\begin{tabular}{|c|c|c|c|c|c|c|c|c|c|c|c|c|c|c|c|c|}
\hline \multirow[b]{2}{*}{ Studies } & \multicolumn{10}{|c|}{ CBT Features } & \multicolumn{2}{|c|}{$\begin{array}{l}\text { Treatment } \\
\text { Modality }\end{array}$} & \multicolumn{2}{|c|}{$\begin{array}{l}\text { Delivery } \\
\text { Method }\end{array}$} & \multicolumn{2}{|c|}{$\begin{array}{c}\text { Delivery } \\
\text { Format }\end{array}$} \\
\hline & $\begin{array}{l}\text { Ed- } \\
\text { uca- } \\
\text { tion }\end{array}$ & $\begin{array}{c}\text { Goal } \\
\text { setting }\end{array}$ & $\begin{array}{c}\text { Iden- } \\
\text { tify } \\
\text { thou } \\
\text {-ghts }\end{array}$ & $\begin{array}{l}\text { Cog- } \\
\text { nitive } \\
\text { re- } \\
\text { struc- } \\
\text { ture }\end{array}$ & $\begin{array}{c}\text { Be- } \\
\text { hav- } \\
\text { ior } \\
\text { acti- } \\
\text { va- } \\
\text { tion }\end{array}$ & $\begin{array}{c}\text { Grade } \\
\text { expo- } \\
\text { sure }\end{array}$ & $\begin{array}{l}\text { Be- } \\
\text { hav- } \\
\text { ioral } \\
\text { ex- } \\
\text { peri- } \\
\text { ment }\end{array}$ & $\begin{array}{l}\text { Stre } \\
\text {-ss } \\
\text { re- } \\
\text { duc- } \\
\text { tion/ } \\
\text { relax- } \\
\text { ation }\end{array}$ & $\begin{array}{c}\text { Re- } \\
\text { lapse } \\
\text { pre- } \\
\text { ven- } \\
\text { tion }\end{array}$ & $\begin{array}{l}\text { Home- } \\
\text { work/ } \\
\text { work- } \\
\text { book }\end{array}$ & $\begin{array}{l}\text { Sin- } \\
\text { gle }\end{array}$ & $\begin{array}{c}\text { Com- } \\
\text { bined/ } \\
\text { supple- } \\
\text { ment }\end{array}$ & $\begin{array}{c}\text { Tele- } \\
\text { phone/ } \\
\text { inter- } \\
\text { net }\end{array}$ & $\begin{array}{l}\text { Face } \\
\text { to } \\
\text { face }\end{array}$ & $\begin{array}{l}\text { Indi- } \\
\text { vid- } \\
\text { ual }\end{array}$ & $\begin{array}{l}\text { Gro- } \\
\text {-up }\end{array}$ \\
\hline $\begin{array}{l}\text { Chalder, } \\
\text { Godfrey, } \\
\text { Ridsdale, } \\
\text { King and } \\
\text { Wessely }\end{array}$ & & & & & & & & & & $\checkmark$ & $\checkmark$ & & & $\checkmark$ & & $\checkmark$ \\
\hline $\begin{array}{c}\text { Currie, } \\
\text { Wilson } \\
\text { and } \\
\text { Curran }\end{array}$ & $\checkmark$ & & & & & & $\checkmark$ & $\checkmark$ & & $\checkmark$ & $\checkmark$ & & & $\checkmark$ & & $\checkmark$ \\
\hline $\begin{array}{c}\text { Flor and } \\
\text { Birbau- } \\
\text { mer }\end{array}$ & & & $\checkmark$ & & & & & $\checkmark$ & & & $\checkmark$ & & & $\checkmark$ & & $\checkmark$ \\
\hline
\end{tabular}




\begin{tabular}{|c|c|c|c|c|c|c|c|c|c|c|c|c|c|c|c|c|}
\hline $\begin{array}{l}\text { Gersh, et } \\
\text { al. [38] }\end{array}$ & & $\checkmark$ & & $\checkmark$ & $\checkmark$ & & $\checkmark$ & $\checkmark$ & & & & $\checkmark$ & & $\checkmark$ & & $\checkmark$ \\
\hline $\begin{array}{l}\text { Kempke, } \\
\text { et al. [35] }\end{array}$ & & & & & & & & & & & & $\checkmark$ & & $\checkmark$ & & $\checkmark$ \\
\hline $\begin{array}{c}\text { Litt \& } \\
\text { Porto } \\
\text { [37] }\end{array}$ & & & & $\checkmark$ & & & & $\checkmark$ & & $\checkmark$ & & $\checkmark$ & & $\checkmark$ & & \\
\hline $\begin{array}{l}\text { Ljotsson, } \\
\text { et al. [51] }\end{array}$ & $\checkmark$ & & $\checkmark$ & & $\checkmark$ & $\checkmark$ & $\checkmark$ & & & $\checkmark$ & $\checkmark$ & & $\checkmark(\mathrm{I})$ & & $\checkmark$ & \\
\hline $\begin{array}{l}\text { Prins, et } \\
\text { al. }\end{array}$ & $\checkmark$ & & $\checkmark$ & $\checkmark$ & & & & & $\checkmark$ & & $\checkmark$ & & & $\checkmark$ & & \\
\hline $\begin{array}{l}\text { Samwel } \\
\text { et al. [39] }\end{array}$ & & $\checkmark$ & & $\checkmark$ & $\checkmark$ & & & $\checkmark$ & & & & $\checkmark$ & & $\checkmark$ & & $\checkmark$ \\
\hline $\begin{array}{l}\text { Schreurs, } \\
\text { et al. [41] }\end{array}$ & & $\checkmark$ & & $\checkmark$ & & & & & & & & $\checkmark$ & & $\checkmark$ & $\checkmark$ & \\
\hline $\begin{array}{l}\text { Sharpe, } \\
\text { et al. }\end{array}$ & & & $\checkmark$ & & $\checkmark$ & $\checkmark$ & $\checkmark$ & $\checkmark$ & & & & $\checkmark$ & & $\checkmark$ & $\checkmark$ & \\
\hline $\begin{array}{c}\text { Siemons- } \\
\text { ma, et al. } \\
\text { [41] }\end{array}$ & & & $\checkmark$ & $\checkmark$ & $\checkmark$ & & $\checkmark$ & & & & & $\checkmark$ & & $\checkmark$ & $\checkmark$ & \\
\hline $\begin{array}{l}\text { Zonne- } \\
\text { veld, et } \\
\text { al., [52] }\end{array}$ & $\checkmark$ & $\checkmark$ & $\checkmark$ & $\checkmark$ & $\checkmark$ & & $\checkmark$ & $\checkmark$ & $\checkmark$ & & $\checkmark$ & & & $\checkmark$ & & $\checkmark$ \\
\hline Total & $4 / 11$ & $4 / 11$ & $6 / 11$ & $8 / 11$ & $6 / 11$ & $2 / 11$ & $6 / 11$ & $6 / 11$ & $2 / 11$ & $3 / 11$ & $4 / 11$ & $7 / 11$ & $2 / 11$ & $9 / 11$ & $6 / 11$ & $4 / 11$ \\
\hline
\end{tabular}

Note: I=internet; $\mathrm{T}=$ telephone

In all studies, therapists who delivered the CBT intervention were required to have at least a master's level of education and were either trained or accredited for conducting CBT intervention. The integrity of the interventions was monitored using a variety of

\section{Methodology and identification of clinically significant outcomes}

Table 4: Methodology and Clinically Significant Outcome Identification Methods and Results.

\begin{tabular}{|c|c|c|c|c|c|}
\hline Authors & $\begin{array}{l}\text { Design/data } \\
\text { Collection }\end{array}$ & Outcome Measurement & $\begin{array}{c}\text { Clinically Significant } \\
\text { Outcomes Identification } \\
\text { Methods }\end{array}$ & Predictors & Result \\
\hline \multicolumn{6}{|c|}{ Short term effectiveness (immediately after the treatment) } \\
\hline $\begin{array}{c}\text { Gersh et al. } \\
\text { [38] }\end{array}$ & $\begin{array}{l}\text { Pre-post test } \\
\text { Data was collected pre } \\
\text { and post intervention }\end{array}$ & $\begin{array}{l}\text { Brief pain inventory } \\
\text { average pain rating, } \\
\text { Short form health survey } \\
\text { (SF-36 version2) mental } \\
\text { health and physical func- } \\
\text { tioning subscales }\end{array}$ & $\begin{array}{l}\text { 1.Using the score of PSOCQ } \\
\text { as a continuous variable } \\
\text { and investigate the associa- } \\
\text { tion of score change } \\
\text { 2.Allocated the participants } \\
\text { into the } 4 \text { stages of changes } \\
\text { include, precontemplation, } \\
\text { contemplation, action and } \\
\text { maintenance based on the } \\
\text { maximum score. If score } \\
\text { was high in more than one } \\
\text { scale, participants will } \\
\text { be placed in the further } \\
\text { progression stage. } \\
\text { 3.Classified participants } \\
\text { based on the change of } \\
\text { PSOCQ score into progres- } \\
\text { sor (move from precontem- } \\
\text { plation to contemplation, } \\
\text { action and maintenance } \\
\text { and people who remain } \\
\text { in the maintenance stage) } \\
\text { and non-progressors } \\
\text { (regressed or no change of } \\
\text { the stage) }\end{array}$ & $\begin{array}{l}\text { Pain stages of change } \\
\text { questionnaire (PSO- } \\
\text { CQ) }\end{array}$ & $\begin{array}{l}\text { Improvement in mood } \\
\text { rating associated with the } \\
\text { decreased precontempla- } \\
\text { tion }(\mathrm{r}=-.24, \mathrm{p}<0.05) \text { and } \\
\text { increased maintenance } \\
\text { rating }(\mathrm{r}=.29, \mathrm{p}<0.05) \\
\text { Decrease disability rating } \\
\text { associated with increase } \\
\text { rating on action }(\mathrm{r}=.22 \text {, } \\
\mathrm{p}<0.05) \text { and maintenance } \\
(\mathrm{r}=.18, \mathrm{p}<0.05) \text { scales. } \\
\text { High in precontemplation } \\
\text { and low in other subscale } \\
\text { of PSOCQ are less likely to } \\
\text { complete treatment } \\
\text { Progressors showed more } \\
\text { improved of mood (F } \\
(1,117)=9.60, \mathrm{p}<0.01) \text { and } \\
\text { function }(\mathrm{F}(1,117)=4.00, \\
\mathrm{p}<0.01) \text { than } \\
\text { non-progressors } \\
\text { Both progressors and } \\
\text { non-progressors showed } \\
\text { no significant different of } \\
\text { pain change magnitude }(\mathrm{F} \\
(1.117)=0.88, \mathrm{p}<0.05) \\
\text { Treatment groups were } \\
\text { not differ regarding to the } \\
\text { stage of change }\end{array}$ \\
\hline
\end{tabular}




\begin{tabular}{|c|c|c|c|c|c|}
\hline $\begin{array}{l}\text { Kempke, et } \\
\text { al. [35] }\end{array}$ & $\begin{array}{l}\text { Pre-post test } \\
\text { The predictors were } \\
\text { measured at baseline. } \\
\text { The outcome variables } \\
\text { were measured at } \\
\text { completion of the } \\
\text { intervention }\end{array}$ & $\begin{array}{l}\text { Post- Fatigue measured } \\
\text { by } \\
\text { the checklist individual } \\
\text { strength (CIS-20) }\end{array}$ & $\begin{array}{l}\text { The association of the } \\
\text { predictors } \\
\text { at baseline with the level of } \\
\text { outcome variables (fatigue) } \\
\text { at treatment completion } \\
\text { was tested } \\
\text { Participants were classified } \\
\text { into substantial improved } \\
\text { using the } 10 \% \text { reduction of } \\
\text { fatigue (CIS-20) based on } \\
\text { the effect sized (Cohen's d } \\
=0.79 \text { ) }\end{array}$ & $\begin{array}{l}\text { Pre-Fatigue mea- } \\
\text { sured by the checklist } \\
\text { individual strength(- } \\
\text { CIS-20) } \\
\text { Patient's opinions } \\
\text { regarding the cause } \\
\text { of their fatigue } \\
\text { measured by Causal } \\
\text { Attribution list (CAL) } \\
\text { Self-efficacy scale } \\
\text { (SES) } \\
\text { General activity level } \\
\text { index of the Multi- } \\
\text { dimensional Pain } \\
\text { Inventory (MPI) } \\
\text { Function impairment } \\
\text { measured by Func- } \\
\text { tioning Scale (SF-36) } \\
\text { Psychiatric and so- } \\
\text { matic conditions: the } \\
\text { somatization subscale } \\
\text { of the symptom } \\
\text { Checklist-90 (SCL-90) } \\
\text { Severity of depression } \\
\text { measured by the de- } \\
\text { pression subscale of } \\
\text { the Hospital Anxiety } \\
\text { and Depression Scale } \\
\text { (HADS) }\end{array}$ & $\begin{array}{c}\text { High pre-treatment } \\
\text { depression was associated } \\
\text { with high post treatment } \\
\text { fatigue }(\beta=.239, \mathrm{p}<.05) \\
\text { Higher } \\
\text { pre-treatment depression } \\
\text { patients were less likely } \\
\text { to experience fatigue im- } \\
\text { proved after the treatment } \\
\text { (Wald }=5.527, \mathrm{p}=05, \\
\mathrm{OR}=.862,95 \% \mathrm{CI}=.762- \\
.976) .\end{array}$ \\
\hline $\begin{array}{c}\text { Samwel, et al. } \\
\text { [39] }\end{array}$ & Pre-post test design & $\begin{array}{l}\text { Pain intensity: 10- cen- } \\
\text { trimeter visual analogue } \\
\text { scale (VAS) rating for } 7 \\
\text { days } \\
\text { Functional disability: } \\
\text { Dutch version of the } \\
\text { pain disability index } \\
\text { (PDI), measure role of } \\
\text { functioning: family/ } \\
\text { home responsibilities, } \\
\text { recreation, social activ- } \\
\text { ities, occupation, sexual } \\
\text { behavior, self-care, life } \\
\text { supporting activities } \\
\text { Depression: Dutch } \\
\text { version of the symptoms } \\
\text { checklist-90 } \\
\text { Medication Use: calculat- } \\
\text { ed from the actual daily } \\
\text { used of drugs (UDD)/ } \\
\text { amount of drugs needed } \\
\text { to obtain the desired } \\
\text { effect on pain in the gen- } \\
\text { eral population (DDD) }\end{array}$ & $\begin{array}{l}\text { The association between } \\
\text { the baseline predictor vari- } \\
\text { able scores and the change } \\
\text { score of the outcomes from } \\
\text { baseline to completion was } \\
\text { tested. }\end{array}$ & $\begin{array}{c}\text { Avoidance behavior: } \\
\text { passive pain coping } \\
\text { scales retreating and } \\
\text { resting of the pain } \\
\text { coping inventory } \\
\text { (PCI) } \\
\text { Worrying: worrying } \\
\text { scale of the PCI } \\
\text { Fear of pain: adjusted } \\
\text { version of Tampa } \\
\text { scale of Kinesiophobia } \\
\text { Helplessness: } \\
\text { helplessness scale of } \\
\text { the illness cognition } \\
\text { questionnaire (ICQ) } \\
\text { Acceptance: accep- } \\
\text { tance items of ICQ }\end{array}$ & $\begin{array}{l}\text { Higher levels of accep- } \\
\text { tance to significantly } \\
\text { predict larger reduc- } \\
\text { tions of pain intensity in } \\
\text { intervention but not in the } \\
\text { control group }\end{array}$ \\
\hline $\begin{array}{c}\text { Siemonsma, et } \\
\text { al. [42] }\end{array}$ & $\begin{array}{l}\text { Pre-post test } \\
\text { Data was collected } \\
\text { before and at the } \\
\text { completion of the } \\
\text { intervention }\end{array}$ & $\begin{array}{c}\text { Patient-specific } \\
\text { Functioning list (PSFL) } \\
\text { change: the change score } \\
\text { from baseline to treat- } \\
\text { ment completion }\end{array}$ & $\begin{array}{l}\text { Association of the baseline } \\
\text { predictor variable scores } \\
\text { and the change of outcome } \\
\text { variable: PSFL were tested. } \\
\text { Use the criteria of mini- } \\
\text { mum decrease of the PSFL } \\
\text { at least } 18 \mathrm{~mm} \text { to identify } \\
\text { the clinical significant } \\
\text { change }\end{array}$ & $\begin{array}{l}\text { Rational prob- } \\
\text { lem-solving: The rela- } \\
\text { tional problem-solv- } \\
\text { ing (RPS) scale } \\
\text { Discussion skills: } \\
\text { Aggrievedness Scale } \\
\text { of Dutch Personality } \\
\text { questionnaire } \\
\text { Verbal skills: Multi- } \\
\text { cultural Capacity Test } \\
\text { (MCT), Word Relation } \\
\text { Scale (MCT-WR and } \\
\text { World Analogies Scale } \\
\text { (MCT- } \\
\text { WA) }\end{array}$ & $\begin{array}{c}\text { Rational problem-solving } \\
\text { found to be significant pre- } \\
\text { dictor for the change } \\
\text { in physical activity. } \\
\text { Discussion skills and } \\
\text { verbal skills were not } \\
\text { significant. } \\
\text { Rational problem-solving } \\
\text { explained 3.9\% of total } \\
\text { variance. }\end{array}$ \\
\hline
\end{tabular}




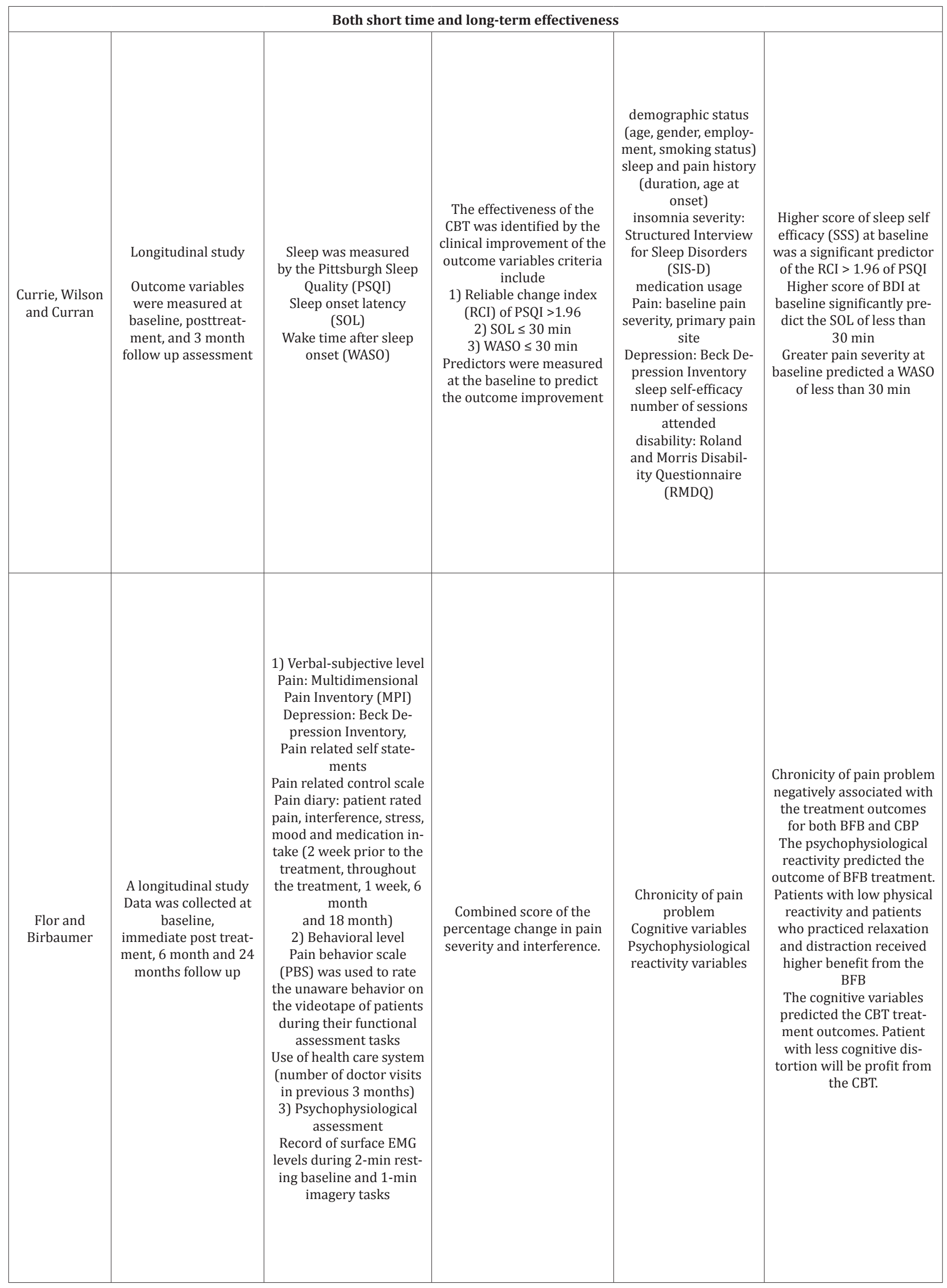




\begin{tabular}{|c|c|c|c|c|c|}
\hline $\begin{array}{l}\text { Litt \& Porto } \\
\text { [37] }\end{array}$ & $\begin{array}{c}\text { Pre, post test } \\
\text { Data was collected at } \\
\text { pre-treatment, treat- } \\
\text { ment completion (6 } \\
\text { weeks) and at weeks } \\
12,24,36 \\
\text { and } 52\end{array}$ & $\begin{array}{l}\text { Pain (multiple pain In- } \\
\text { ventory: MPI) response } \\
\text { at baseline and } 1 \text { year } \\
\text { post treatment }\end{array}$ & $\begin{array}{l}\text { The responders and } \\
\text { non-responders were iden- } \\
\text { tified using the statistic } \\
\text { methods, Growth Mixture } \\
\text { modeling (GMM) to inves- } \\
\text { tigate the pattern of MPI } \\
\text { pain changes over times. }\end{array}$ & $\begin{array}{l}\text { Demographic infor- } \\
\text { mation (sex, age, race, } \\
\text { education, employ- } \\
\text { ment status, marital } \\
\text { status, income) } \\
\text { Pain and TMJ pathol- } \\
\text { ogy variables: patient } \\
\text { history question- } \\
\text { naire of Research } \\
\text { Diagnostic Criteria } \\
\text { for temporomandib- } \\
\text { ular disorders (RDC/ } \\
\text { TMJ): Axis I Physical } \\
\text { disorder and Axis II } \\
\text { psychosocial axis } \\
\text { Psychiatric } \\
\text { symptoms: } \\
\text { 1)Depression (Center } \\
\text { of epidemiological } \\
\text { studies depression } \\
\text { scale (CESD) } \\
\text { 2)Anxiety: state-trait } \\
\text { anxiety inventory } \\
\text { 3)Somatization: } \\
\text { 12-item somatiza- } \\
\text { tion subscale of the } \\
\text { symptoms checklist } \\
\text { 90-revised (SCL-9-R) } \\
\text { Cognitive constructs: } \\
\text { 1) Readiness to en- } \\
\text { gage in self- manage- } \\
\text { ment treatment: the } \\
\text { pain stages of change } \\
\text { questionnaire } \\
\text { 2)Pain management } \\
\text { self- efficacy: Chronic } \\
\text { pain Self-efficacy } \\
\text { scale (CPSS) }\end{array}$ & $\begin{array}{l}\text { Treatment conditions, and } \\
\text { catastrophizing change } \\
\text { were significant predictors } \\
\text { of the member in the high } \\
\text { pain, treatment responder } \\
\text { group }\end{array}$ \\
\hline $\begin{array}{c}\text { Ljotsson et al. } \\
\text { [53] }\end{array}$ & $\begin{array}{l}\text { Randomized con- } \\
\text { trolled crossed over } \\
\text { design }\end{array}$ & $\begin{array}{l}\text { Gastrointestinal symp- } \\
\text { tom rating scale-IBS } \\
\text { version (GSRS-IBS) }\end{array}$ & $\begin{array}{l}\text { The associations of } \\
\text { the baseline predictor } \\
\text { variables scores and the } \\
\text { GSRS-IBS scores at the post } \\
\text { treatment, follow up scores } \\
\text { were tested }\end{array}$ & $\begin{array}{c}\text { Psychological distress } \\
\text { measured by } \\
\text { Mini-International } \\
\text { Neuropsychiatric } \\
\text { Interview (MINI) } \\
\text { Depression measured } \\
\text { by Montgomery } \\
\text { Asberg Depression } \\
\text { Rating Scale-Self } \\
\text { report (MADRS-S) } \\
\text { Severity of somatic } \\
\text { symptoms measured } \\
\text { by Somatization } \\
\text { subscale of Symptoms } \\
\text { Checklist-90 (SCL- } \\
\text { SOM) } \\
\text { Gastrointestinal } \\
\text { symptom-specific } \\
\text { anxiety } \\
\text { (GSA) and related } \\
\text { constructs measured } \\
\text { by Visceral Sensitivity } \\
\text { Index (VSI) } \\
\text { Catastrophizing mea- } \\
\text { sured by Catastroph- } \\
\text { izing subscale of } \\
\text { Coping Strategies } \\
\text { Questionnaire } \\
\text { Anxiety measured by } \\
\text { Anxiety Sensitivity } \\
\text { Index (ASI) } \\
\text { Disability } \\
\text { Quality of life mea- } \\
\text { sured by Irritable } \\
\text { Bowel Syndrome } \\
\text { Quality of Life (IBS- } \\
\text { QOL) }\end{array}$ & $\begin{array}{l}\text { When control for the pre- } \\
\text { treatment symptoms, no } \\
\text { significant predictors }\end{array}$ \\
\hline
\end{tabular}




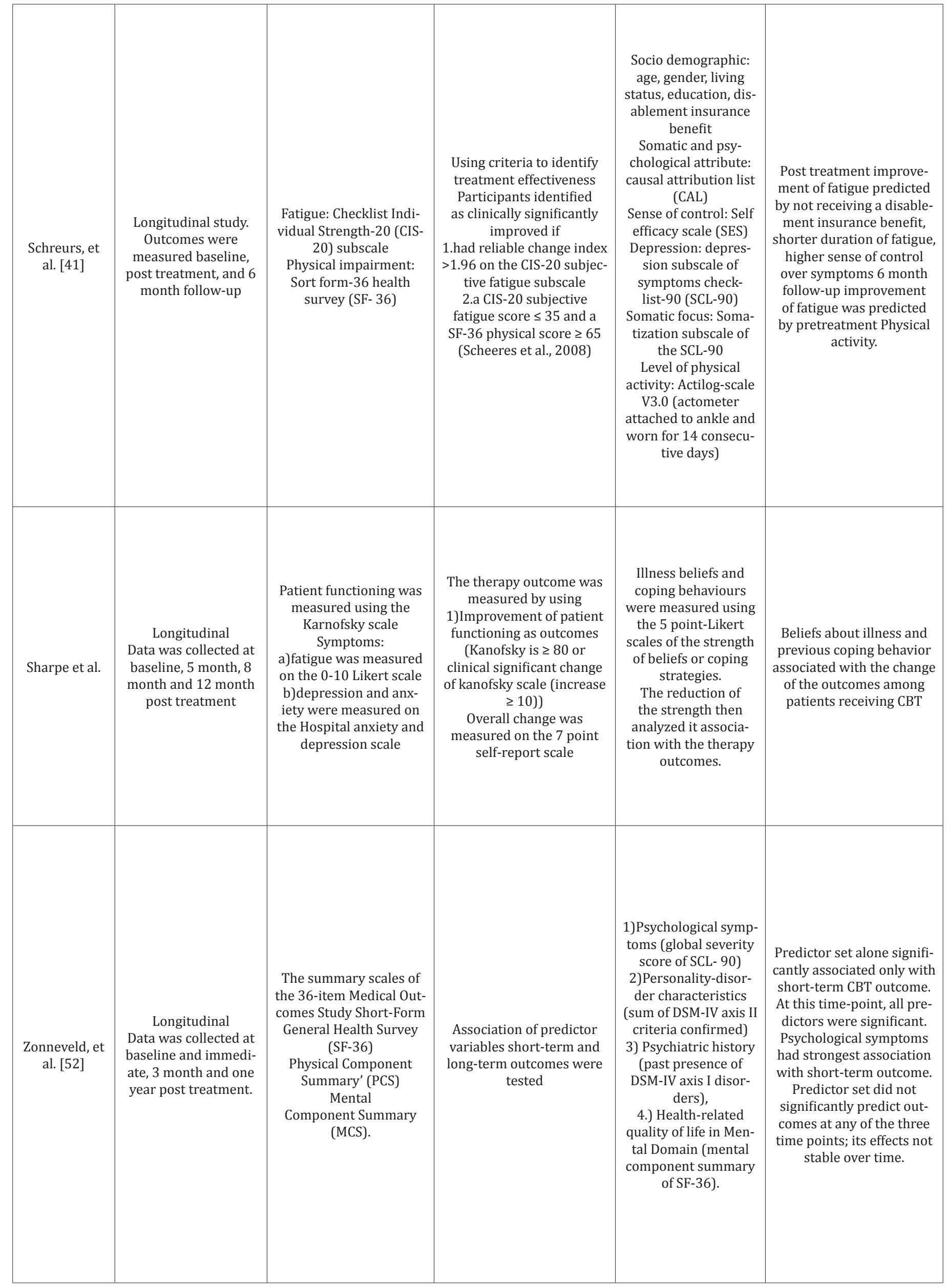




\begin{tabular}{|c|c|c|c|c|c|}
\hline \multicolumn{6}{|c|}{ Only long term effectiveness of CBT (more than one month post treatment) } \\
\hline $\begin{array}{l}\text { Chalder, God- } \\
\text { frey, Ridsdale, } \\
\text { King and } \\
\text { Wessely }\end{array}$ & $\begin{array}{l}\text { Longitudinal study } \\
\text { Outcome variables } \\
\text { were measured at } \\
\text { baseline and } 6 \text { month } \\
\text { post treatment }\end{array}$ & $\begin{array}{l}\text { Fatigue is measured by } \\
\text { the fatigue question- } \\
\text { naire. Fatigue score } \\
\text { range from } 0 \text { to } 11\end{array}$ & $\begin{array}{c}\text { Patients scored fatigue } \\
\text { of } 4 \text { or higher at } 6 \text { month } \\
\text { post treatment is consider } \\
\text { fatigue case }\end{array}$ & $\begin{array}{l}\text { 1)Disability is } \\
\text { measured by social } \\
\text { adjustment scale } \\
\text { 2)Anxiety and depres- } \\
\text { sion are measured } \\
\text { using the Hospital } \\
\text { Anxiety and Depres- } \\
\text { sion Scale } \\
\text { 3) Illness perception } \\
\text { was measured on } \\
\text { the how individual } \\
\text { perceived their illness } \\
\text { which scored of } \\
\text { symptoms quality on } \\
\text { causes, consequences, } \\
\text { timeline and control- } \\
\text { lability } \\
\text { 4) Attribution is } \\
\text { measured by using } 5 \\
\text { response options for } \\
\text { the contribution of } \\
\text { physical or psycholog- } \\
\text { ical on their illness }\end{array}$ & $\begin{array}{c}\text { Among fatigue case at } 6 \\
\text { month post CBT. } \\
\text { no significant association } \\
\text { of demographics variables } \\
\text { and outcome variable at } 6 \\
\text { months } \\
\text { Perception of the per- } \\
\text { sistent of illness, belief } \\
\text { that state of illness caused } \\
\text { by physical, poor social ad- } \\
\text { justment were associated } \\
\text { with fatigue }\end{array}$ \\
\hline Prins, et al & $\begin{array}{l}\text { Longitudinal study } \\
\text { Outcome variables } \\
\text { were measured at } \\
\text { baseline, immediate } \\
\text { follow up (8 } \\
\text { month) and 14- month } \\
\text { follow up }\end{array}$ & $\begin{array}{c}\text { Fatigue severity: a } \\
\text { subscale of checklist } \\
\text { individual strength } \\
\text { Functional impairment: } \\
\text { the sickness impact } \\
\text { profile } \\
\text { Performance status: the } \\
\text { Karnofsky performance } \\
\text { status scale } \\
\text { Quality of life: the visual } \\
\text { analogue scale of the } \\
\text { EuroQol } \\
\text { Hours working in a job } \\
\text { Self-rated improvement } \\
\text { Physical activity: actom- } \\
\text { eter (a motion-sensing } \\
\text { device attached the } \\
\text { ankle and worn for } 12 \\
\text { days }\end{array}$ & $\begin{array}{c}\text { Patients will be classified } \\
\text { as showing clinically im- } \\
\text { provement of both of these } \\
\text { criteria were met } \\
\text { 1.The reliable change index } \\
\text { of fatigue score (RCI > 1.64, } \\
\text { p <0.05) } \\
\text { 2.Patients' fatigue score } \\
\text { move from the range of } \\
\text { CFS to the range of healthy } \\
\text { control (the cut-off score } \\
\text { of } 36 \text { or lower for healthy } \\
\text { control fatigue) }\end{array}$ & $\begin{array}{l}\text { Psychological well- } \\
\text { being: the symptom } \\
\text { checklist } 90 \\
\text { Self efficacy: self-effi- } \\
\text { cacy scale } \\
\text { Somatic attributions: } \\
\text { The causal attribution } \\
\text { list } \\
\text { Focusing on bodily } \\
\text { symptoms: the } \\
\text { subscale somatiza- } \\
\text { tion of the symptoms } \\
\text { checklist } 90\end{array}$ & $\begin{array}{l}\text { In CBT group, patients } \\
\text { who have low sense of } \\
\text { control and have passive } \\
\text { activity pattern experi- } \\
\text { enced less improvement of } \\
\text { fatigue severity at immedi- } \\
\text { ate after treatment. } \\
\text { In CBT group, patients } \\
\text { who have high level } \\
\text { of focusing on bodily } \\
\text { symptoms showed little } \\
\text { improve of the } \\
\text { treatment outcomes. }\end{array}$ \\
\hline
\end{tabular}

(Table 4) describes the study design, outcome variables, clinically significant outcomes identification methods, predictors, and results. Four studies only investigated the immediate effectiveness of CBT by measuring pre- and post-treatment outcomes [38,39]. Seven studies evaluated both short- and longterm outcomes by evaluating participants for up to one year following the intervention [40]. Methods used to identify the success or responsiveness to the intervention were varied. Gersh et al. [41] identified criteria to classify participants into different groups based on the stage of change scores. Five studies classified participants into clinical improvement and nonclinical improvement groups using the cut-off score of outcome variables such as fatigue, function, and depression. One study used a sophisticated statistical method to analyze the pattern of change in outcomes over time and then used it as a criterion to group responders to the intervention Litt \& Porto [42]. Ten other studies investigated the predictability of the predictors on either the outcome variables post-treatment.

\section{Psychosocial predictors of CBT success}

Most of the predictors in these 11 articles were psychosocial. The predictors included: states of change Gersh et al. [41,42] post-intervention psychiatric and somatic conditions Kempke et al. Litt \& Porto, Ljotsson, Andersson, et al., 2013; Zonneveld et al., self-efficacy Kempke et al., Schreurs et al., behaviors such as avoidance, worrying, fear, helplessness, and acceptance Samwel et al., skills such as problem solving, discussion, and verbal skills Siemonsma et al., therapeutic alliance Applebaum et al., and caregiver participation Dobkin et al. Only one study investigated the functional brain circuit in association with the response to the CBT intervention Falconer et al.

\section{Discussion}

All 11 studies determined that patients with physical illnesses benefit from CBT in both the short and long term. However, these results also found that not all participants receive the same level of benefit. Several factors may influence the effectiveness of a CBT intervention. First, although the 11 articles used the term CBT intervention, they differed in their intervention components, treatment modalities, and delivery methods. Although evidence suggests that the phone/internet-based CBT intervention and a face-to-face CBT intervention can have comparable effects, [43-47] the intervention components of CBT used in these studies varied (Table 3). The recommended intervention features for CBT as an adjunctive treatment in chronic physical illnesses include cognitive 
intervention (e.g., goal setting, education, cognitive restructuring, identifying thoughts/beliefs) and behavior intervention (e.g., behavioral activation, grade exposure, behavioral experiments and pacing, stress reduction training, and relapse prevention; Halford \& Brown, 2009). To ensure that patients use these techniques, homework or workbook assignments are needed. However, the CBT studies described in these articles do not include all of these features (Table 3). Second, social support may have had a major influence on the effectiveness of studies that compared group sessions to individual sessions. Third, the number of sessions and time spent for each session varied widely among the reviewed articles. Finally, 7 of the 11 studies used CBT as an adjunct treatment with other interventions. These differences of treatment modality and methods may have led to differences in outcomes.

Methods used to identify the success of CBT were inconsistent among the articles. These Two main methods were used by the reviewed articles include the use of criteria to classify the participants into treatment responders and non-responders and the use outcome variables at the treatment complication or the level of outcome change at completion from baseline. These inconsistencies can have a major impact on the identification of predictors and make it difficult to determine who will benefit from CBT intervention. The standard criteria or expected outcomes for the CBT intervention should be developed to identify the effectiveness.

Consistent with McCracken \& Turk's (2002) review article on the predictors of outcomes of CBT in patients with chronic pain, we found that most of the significant predictors were psychosocial predictors. Unlike McCracken and Turk's review, however, our results showed that the patients' level of readiness to change, acceptance, rational problem-solving, and depression predicted improvement of the outcomes. These outcomes included a shortterm effectiveness of the CBT intervention on pain, fatigue, and physical activity. Interestingly, these predictors often overlapped or were associated with each other. For example, the stage of readiness to change "contemplation," requires persons to think rationally about their situation and its solution, which can overlap with rational problem solving. The association among states of readiness to change, acceptance, and rational-problem solving with depression were reported in three of the studies [48-50]. These associations and overlapping outcome predictors could influence the results of a study. Each of these predictors was studied separately and no study investigated all of the predictors in one disease phenomenon.

To investigate the predictors that help identify responders to CBT intervention, seven studies identified predictors of both immediate and long-term effectiveness [51]. However, results among the studies were inconsistent, with different significant predictors for immediate and long-term outcomes. In patients with chronic fatigue syndrome, for example, physical activity and a feeling of control over symptoms predicted an immediate outcome improvement, but disability benefit was a predictor for outcomes at
6 months Schreurs et al. [52]. For patients with unexplained physical symptoms, the mental component was a significant outcome predictor of CBT at 3 months, but not significant for the immediate and long-term (1 year after the intervention) outcomes. Using a sophisticated statistical technique, Growth Mixture Modeling, Litt and Porto demonstrated that the change of catastrophizing, persons' negative evaluation and attention on a specific event, predicted the member of CBT responders' group. Two studies found consistent significant predictors of immediate and long-term outcomes. Dopkin et al. discovered that caregiver participation was the only significant predictor of the CBT responders at the end of the intervention and one month after. Applebaum et al. [53] determined that the therapeutic alliance significantly predicted immediate outcomes and outcomes one year after the intervention. In one study there was no significant outcome predictor for CBT in people with irritable bowel syndrome. A number of studies reported the biological predictors of the CBT outcomes in the psychological disorder [54-56]. Moreover, a recent study reported the expression change of genes associated with mood states in major depression patients Keri, Szabo, \& Kelemen [57,58]. This information will not only help identify the biological mechanism associated with the CBT effectiveness but also identity person potentially will benefit from the intervention. Based on the articles reviewed, only one study investigated the association of physiological outcome predictors of CBT outcomes posttraumatic stress disorder in civilian trauma. The study result suggested the neural activation pattern of the left-lateralized front striatal inhibitory control associated with the response to CBT. This finding suggested future research should examine the biological pathways or mechanisms associated with CBT outcomes.

This rigorous, targeted review of 11 randomized control trials adds to the field of knowledge on CBT outcome predictors for physical illnesses. The results can be used as a guide for future researchers in investigating CBT intervention outcomes predictors in people with chronic physical illnesses, especially physiological and biological predictors. Furthermore, psychological predictors such as acceptance, therapeutic alliance, self-efficacy, physical ability, and depression should be tested for their predictability among people with different physical illnesses. Finally, a standardized guideline of CBT intervention with common components applicable to physical illnesses should be developed and tested.

\section{Limitations}

The sample size was small because our search was limited to randomized control trials that included an investigation of the outcome predictors. Therefore, several comparable but nonrandomized trials were not reviewed. Additionally, the review only included physiological illnesses, so numbers of studies investigating biological predictors associated with CBT outcomes on depression and most other psychological disorders were not included.

\section{Acknowledgement}

This abstract was published under the title "Predictors of Cognitive Behavioral Therapy Response in Chronic Diseases: 
Integrative Review" as a proceeding abstract of the 2015 Asia 2015 Asian American/Pacific Islander Nurses Association Conference.

\section{Conflict of interest}

No conflict of interest.

\section{References}

1. Ward B W, Shchiller J S, Goodman R A (2014) Multiple chronic conditiona among US adults: A 2012 update. Prev Chronic Dis 11: E62.

2. Whittemore R, Dixon J (2008) Chronic illness: the process of integration. J Clin Nurs 17(7B): 177-187.

3. Beck J S (1995) Cognitive Behavioral Therapy Basics and Beyond: Guilford Press, USA.

4. Halford J, Brown T (2009) Cognitive-behavioural therapy as an adjunctive treatment in chronic physical illness. Advances in Psychiatric Treatment 15: 306-317.

5. Butler A C, Chapman J E, Forman E M, Beck A T (2006) The empirical status of cognitive-behavioral therapy: a review of meta-analyses. Clin Psychol Rev 26(1): 17-31.

6. Turk D C (2003) Cognitive-behavioral approach to the treatment of chronic pain patients. Reg Anesth Pain Med 28(6): 573-579.

7. Watanabe N, Furukawa T A, Chen J, Kinoshita Y, Nakano Y, et al. (2010) Change in quality of life and their predictors in the long-term follow-up after group cognitive behavioral therapy for social anxiety disorder: a prospective cohort study. BMC Psychiatry, 10: 81.

8. Wilkinson P (2013) Cognitive behavioural therapy with older people. Maturitas 76(1): 5-9.

9. Thomas E M, Weiss S M (2000) Nonpharmacological interventions with chronic cancer pain in adults. Cancer Control 7(2): 157-164.

10. Dobkin RD, Menza M, Allen LA, Gara M A, Mark MH, et al. (2011) Cognitive-behavioral therapy for depression in Parkinson's disease: A randomized, controlled trial. Am J Psychiatry 168(10): 1066-1074.

11. Welschen LMC, van Oppen P, Bot SDM, Kostense PJ, Dekker JM, et al. (2013) Effects of a cognitive behavioural treatment in patients with type 2 diabetes when added to managed care; A randomised controlled trial. J Behav Med 36(6): 556-566.

12. Wagner GJ, Kanouse DE, Golinelli D, Miller LG, Daar ES, et al. (2006) Cognitive-behavioral intervention to enhance adherence to antiretroviral therapy: arandomized controlled trial (CCTG 578). AIDS 20(9): 12951302.

13. Sinclair VG, Wallston KA (2001) Predictors of improvement in a cognitive-behavioral intervention for women with rheumatoid arthritis. Ann Behav Med 23(4): 291-297.

14. Inouye J, Flannelly L, Flannelly K J (2001) The effectiveness of selfmanagement training for individuals with HIV/AIDS. J Assoc Nurses AIDS Care 12(2): 73-84.

15. Sinclair KA, Makahi EK, Shea Solatorio C, Yoshimura SR, Townsend CK, et al. (2013) Outcomes from a diabetes self-management intervention for Native Hawaiians and Pacific People: Partners in Care. Ann Behav Med 45(1): 24-32.

16. Safren SA, Cleirigh OC, Tan JY, Raminani SR, Reilly LC, et al. (2009) A randomized controlled trial of cognitive behavioral therapy for adherence and depression (CBT-AD) in HIV-infected individuals. Health Psychol 28(1): 1-10.

17. Thorndike F P, Ritterband L M, Gonder Frederick L A, Lord H R, Ingersoll $\mathrm{K} \mathrm{S}$, et al. (2013) A randomized controlled trial of an internet intervention for adults with insomnia: Effects on comorbid psychological and fatigue symptoms. J Clin Psychol 69(10): 1078-1093.

18. van Beugen S, Ferwerda M, Hoeve D, Rovers M M, Spillekom van Koulil S, et al. (2014) Internet-based cognitive behavioral therapy for patients with chronic somatic conditions: a meta-analytic review. J Med Internet Res 16(3): e88.
19. Barrett P, Farrell L, Dadds M, Boulter N (2005) Cognitive-behavioral family treatment of childhood obsessive-compulsive disorder: longterm follow-up and predictors of outcome. J Am Acad Child Adolesc Psychiatry 44(10): 1005-1014.

20. Rufer M, Fricke S, Moritz S, Kloss M, Hand I (2006) Symptom dimensions in obsessive- compulsive disorder: prediction of cognitive-behavior therapy outcome. Acta Psychiatr Scand 113(5): 440-446.

21. Rufer M, Hand I, Alsleben H, Braatz A, Ortmann J, et al. (2005) Longterm course and outcome of obsessive-compulsive patients after cognitive-behavioral therapy in combination with either fluvoxamine or placebo: a 7-year follow-up of a randomized double- blind trial. Eur Arch Psychiatry Clin Neurosci 255(2): 121-128.

22. Sil S, Arnold L M, Lynch Jordan A, Ting T V, Peugh J, et al. (2014) Identifying treatment responders and predictors of improvement after cognitive- behavioral therapy for juvenile fibromyalgia. Pain 155(7): 1206-1212.

23. van Koulil S, van Lankveld W, Kraaimaat F W, van Helmond T, Vedder A, et al. (2008) Tailored cognitive-behavioral therapy for fibromyalgia: two case studies. Patient Educ Couns 71(2): 308-314.

24. Bernardy K, Fuber N, Kollner V, Hauser W (2010) Efficacy of cognitivebehavioral therapies in fibromyalgia syndrome-a systematic review and metaanalysis of randomized controlled trials. J Rheumatol 37(10): 1991-2005.

25. Campbell C L, Campbell L C (2012) A systematic review of cognitive behavioral interventions in advanced cancer. Patient Educ Couns 89(1): 15-24.

26. Inouye J, Braginsky N, Kataoka Yahiro M (2011) Randomized clinical trials of self-management with Asian/Pacific Islanders. Clin Nurs Res 20(4): 366-403.

27. Cislak A, Safron M, Pratt M, Gaspar T, Luszczynska A (2012) Familyrelated predictors of body weight and weight-related behaviours among children and adolescents: a systematic umbrella review. Child Care Health Dev 38(3): 321-331.

28. Zantvoord JB, Diehle J, Lindauer RJ (2013) Using neurobiological measures to predict and assess treatment outcome of psychotherapy in posttraumatic stress disorder: systematic review. Psychother Psychosom 82(3): 142-151.

29. Jong E, Oudhoff L A, Epskamp C, Wagener M N, van Duijn M, et al. (2010) Predictors and treatment strategies of HIV-related fatigue in the combined antiretroviral therapy era. AIDS 24(10): 1387-1405.

30. Mc Cracken LM, Turk DC (2002) Behavioral and cognitive-behavioral treatment for chronic pain: outcome, predictors of outcome, and treatment process. Spine (Phila Pa 1976) 27(22): 2564-2573.

31. de Rooij A, Roorda LD, Otten RH, van der Leeden M, Dekker J, et al. (2013) Predictors of multidisciplinary treatment outcome in fibromyalgia:a systematic review. Disabil Rehabil 35(6): 437-449.

32. Jadad A R, Carroll D, Moore A, Mc Quay H (1996) Developing a database of published reports of randomised clinical trials in pain research. Pain 66(2-3): 239-246.

33. Applebaum AJ, Du Hamel K N, Winkel G, Rini C, Greene PB, et al. (2012) Therapeutic alliance in telephone-administered cognitive-behavioral therapy for hematopoietic stem cell transplant survivors. J Consult Clin Psychol 80(5): 811-816.

34. Dobkin RD, Rubino JT, Allen LA, Friedman J, Gara MA, et al. (2012) Predictors of treatment response to cognitive-behavioral therapy for depression in Parkinson's disease. J Consult Clin Psychol 80(4): 694-699.

35. Kempke S, Goossens L, Luyten P, Bekaert P, Van Houdenhove B, et al. (2010) Predictors of outcome in a multi-component treatment program for chronic fatigue syndrome. J Affect Disord 126(1-2): 174-179.

36. Falconer E, Allen A, Felmingham KL, Williams LM, Bryant RA (2013) Inhibitory neural activity predicts response to cognitive-behavioral therapy for posttraumatic stress disorder. J Clin Psychiatry 74(9): 895901. 
37. Litt MD, Porto FB (2013) Determinants of pain treatment response and nonresponse: identification of TMD patient subgroups. J Pain 14(11): 1502-1513.

38. Gersh E, Arnold C, Gibson SJ (2011) The relationship between the readiness for change and clinical outcomes in response to multidisciplinary pain management. Pain Med 12(1): 165-172.

39. Samwel HJ, Kraaimaat FW, Crul BJ, van Dongen RD, Evers AW (2009) Multidisciplinary allocation of chronic pain treatment: effects and cognitive-behavioural predictors of outcome. Br J Health Psychol 14(Pt 3): 405-421.

40.Zonneveld LN, van t Spijker A, Passchier J, van Busschbach JJ, Duivenvoorden HJ (2009) The effectiveness of a training for patients with unexplained physical symptoms: protocol of a cognitive behavioral group training and randomized controlled trial. BMC Public Health 9: 251.

41. Schreurs KM, Veehof MM, Passade L, Vollenbroek Hutten MM (2011) Cognitive behavioural treatment for chronic fatigue syndrome in a rehabilitation setting: effectiveness and predictors of outcome. Behav Res Ther 49(12): 908-913.

42. Siemonsma PC, Stuvie I, Roorda LD, Vollebregt JA, Lankhorst GJ, et al. (2011) Best candidates for cognitive treatment of illness perceptions in chronic low back pain: results of a theory-driven predictor study. J Rehabil Med 43(5): 454-460.

43. Andersson G (2012) Guided internet treatment for anxiety disorders. As effective as face-to-face therapies? Stud Health Technol Inform 181: 3-7.

44. Cuijpers P, van Straten A, Andersson G (2008) Internet-administered cognitive behavior therapy for health problems: a systematic review. J Behav Med 31(2): 169-177.

45. Ghahari S, Packer T (2012) Effectiveness of online and face-to-face fatigue self-management programmes for adults with neurological conditions. Disabil Rehabil 34(7): 564-573.

46. Kiropoulos LA, Klein B, Austin DW, Gilson K, Pier C, et al. (2008) Is internet-based CBT for panic disorder and agoraphobia as effective as face-to-face CBT? J Anxiety Disord 22(8): 1273-1284.

47.van Ballegooijen W, Cuijpers P, van Straten A, Karyotaki E, Andersson $G$, et al. (2014) Adherence to Internet-Based and Face-to-Face Cognitive Behavioural Therapy for Depression: A Meta-Analysis. PLoS One 9(7): e100674.
48. Grossman P, Tiefenthaler Gilmer U, Raysz A, Kesper U (2007) Mindfulness training as an intervention for fibromyalgia: evidence of postintervention and 3-year follow-up benefits in well- being. Psychother Psychosom 76(4): 226-233.

49. Henningsen P, Zimmermann T, Sattel H (2003) Medically unexplained physical symptoms, anxiety, and depression: a meta-analytic review. Psychosom Med 65(4): 528-533.

50. Lewis CC, Simons AD, Silva SG, Rohde P, Small DM, et al. (2009) The role of readiness to change in response to treatment of adolescent depression. J Consult Clin Psychol 77(3): 422-428.

51. Ljotsson B, Andersson E, Lindfors P, Lackner J M, Gronberg K, et al. (2013) Prediction of symptomatic improvement after exposure-based treatment for irritable bowel syndrome. BMC Gastroenterol 13(1): 160.

52. Zonneveld LN, van Rood YR, Kooiman CG, Timman R, van t Spijker A, et al. (2012) Predicting the outcome of a cognitive-behavioral group training for patients with unexplained physical symptoms: a one-year follow-up study. BMC Public Health 12: 848.

53. Ljotsson B, Hesser H, Andersson E, Lindfors P, Hursti T, et al. (2013) Mechanisms of change in an exposure-based treatment for irritable bowel syndrome. J Consult Clin Psychol 81(6): 1113-1126.

54. Gendall KA, Joyce PR, Carter FA, McIntosh VV, Bulik CM (2003) Thyroid indices and treatment outcome in bulimia nervosa. Acta Psychiatr Scand 108(3):190-195.

55. Kobayashi K, Shimizu E, Hashimoto K, Mitsumori M, Koike K, et al. (2005) Serum brain-derived neurotrophic factor (BDNF) levels in patients with panic disorder: as a biological predictor of response to group cognitive behavioral therapy. Prog Neuropsychopharmacol Biol Psychiatry 29(5): 658-663.

56. Ritchey M, Dolcos F, Eddington K M, Strauman T J, Cabeza R (2011) Neural correlates of emotional processing in depression: changes with cognitive behavioral therapy and predictors of treatment response. J Psychiatr Res 45(5): 577-587.

57. Keri S, Szabo C, Kelemen O (2014a) Blood biomarkers of depression track clinical changes during cognitive-behavioral therapy. J Affect Disord 164: 118-122.

58. Keri S, Szabo C, Kelemen O (2014b) Expression of Toll-Like Receptors in Peripheral Blood Mononuclear Cells and Response to CognitiveBehavioral Therapy in Major Depressive Disorder. Brain Behav Immun 40: $235-243$. 